\title{
Correspondences Between Linkage Maps and Salivary Chromosome Structure, as Illustrated in the Tip of Chromosome 2R of Drosophila melanogaster
}

By

Calvin B. Bridges

Carnegie Institution of Washington, and the Wm. G. Kerckhoff Biological Laboratories, California Institute of Technology, Pasadena, California

\section{Introduction}

During the past two years the emphasis in the Drosophila work on the structure and workings of the germ plasm has shifted from analysis by breeding methods to analysis by microscopical observations. Before the importance of the salivary chromosomes was pointed out by Painter (Painter 1933), more could be learned about the distribution and behavior of chromosomes and about their internal differentiation by "counting flies" than by looking at the germ cells themselves. Genetical analysis, through studies of mutation, linkage relations and of aberrations such as non-disjunction, inversion, deficiency and translocation, forged far ahead of the checking that was done through examination of the tiny metaphase chromosomes. But it must not be forgotten that it was this sound foundation, with the establishment of the serial order of the genes within the several chromosomes and with an approximation to their real positions in those chromosomes, that gave instant meaning to the seriation of bands along the salivary chromosomes.

The standard maps of the normal salivary chromosomes (Painter 1934, 1935; Bridges 1935) have been indispensable in analyzing the interrelations between the chromosome sub-structures and the linkage maps. But, although correct so far as they go and satisfactory for general orientation, they are inadequate for the precision needed for close point-to-point homologies. A revision of the salivary maps is now under way which will show approximately half again as many bands and will represent more correctly their relative intensities, individual characteristics and their nearly universal appearance in pairs as "doublets".

The correspondences between the cross-banding, the structural features and the distances along salivary chromosomes with the loci, regions and distances along the linkage maps have been studied in detail by use of the right end of chromosome $2 \mathrm{R}$ of Drosophila melano- 
gaster. Homologous regions and points in the two seriations of loci and banding have been established and local differences and peculiarities in the frequencies both of crossing-over and of mutation have been demonstrated. The interconnections have been found through study of the breakage points of five inversions, two translocations and four deficiencies all having one or both breaks within the short section from near the locus of "humpy" to the tip of $2 \mathrm{R}$. In this same region several other aberrations are known and when they also have been analyzed the correspondences should be still finer in grain.

\section{The loci of mutations and their spacing on the linkage map}

Linkage maps are constructed from genetical data on the frequencies of recombination when two or more pairs of alternative characters are involved in the same cross-preferably the "backcross" or "test-cross" The test-cross is the type of mating in which a multiple heterozygote is crossed to the corresponding multiple recessive, and is so called because the progeny are in classes which are a direct replica (aside from differential mortality) of the gametic series of the heterozygous parent. For example, if the recessive brown eye is crossed to the recessive speck and the wild-type doubly heterozygous female $(\mathrm{bw}+/+\mathrm{sp}$ ) is tested by the double recessive bw sp male the test-cross progeny are found (averages of many trials) to be: $\mathrm{bw}=48.75 \%, \mathrm{sp}=48.75 \%$, bw sp $=1.25 \%$ and wild-type $=$ $1.25 \%$. The new combinations total $2.5 \%$. Recombination of linked characters is brought about by crossing-over of the chromosome threads at some point between the loci of the two mutants. Hence for each recombination one crossover must have occurred. But if the loci are far apart two separate crossovers may occur between them and the second would restore the original combination. Thus, for short intervals percentages of recombination are directly equal to cases of crossing over per average hundred gametes tested, but for longer intervals a table of experimentally determined multiple crossing-over frequencies is needed to convert recombination percentages into crossing-over units or linkage-map distances.

In the upper line of Figure 1 is given the linkage map of the extreme right end of chromosome $2 \mathrm{R}$. This map is revised according to the latest information derived from much unpublished work. These mutants are briefly described below, in alphabetical order of the symbols of the loci, giving symbol, locus name, finder, date of discovery, chromosome and locus reference to publications and figures, facts concerning origin, description of phenotype and associated characteristics. Finally is given a valuation or "Rank" designation, 
RK 1 denoting the most useful loci, RK 2 those nearly as good, RK 3 good mutants not accurately mapped, RK 4 poor mutant phenotypes and RK 5 poor phenotypes not accurately mapped. The mutants Minute-1² and Minute-33a were found and located by Dr. J. Schultz, who also determined that the locus of minus is to the right of brown.

a-arc. C. B. Bridges, May 24, '12. 2-99.2. Pub. Bridges and Morgan 1919; fig. 4, plate 7 . Wings broader, bent downward in slight even arc and edges drawn down to diamond shape. Crossveins closer together (ratio crossvein length : inter-cv dist. $=1: 1.9$ ). Classification perfect after practice. RK 2 .

abb-abbreviated. C. B. Bridges, Apr. 6, '28. 2-105.5. Unpublished. Macrochaetae smaller, especially posterior scutellars. Hatches slightly later than normal. Viability only slightly reduced. Classification hard and uncertain in early hatches, improves with age of culture. Three intensifiers found, of which "shrunken" (2-2.3) confers perfect classification. RK 4; with shr, RK 2.

ba-balloon. T. H. Morgan, Nov. '10. 2-107.4. Pub. Bridges and Morgan 1919 ; fig. 1, plate 7 . Wings at first infiated with blood, leaving blisters and vesicles. Venation weak; straggly, plexus-like. Wings smaller, warped, discolored, divergent. Sensitive to temperature: above $25^{\circ} \mathrm{RK} 4$; $19^{\circ}$ or below, classification easy, RK 2 ; ba/Px, below $19^{\circ}$, RK 2 .

bs-blistered. C. B. Bridges, Nov. 16 '11. 2-107.3. Pub. Bridges and Morgan 1919; text-fig. 74. Wings blistered, smaller, pointed. Venation thicker, plexus-like, especially branches from and parallel to L5 beyond CV2, semidominant "free-vein effect" here. Eye-color softened. Sensitive to temp.: at $25^{\circ} \mathrm{RK} 4$; at $19^{\circ}$ and below, RK2. (More extreme allel $=\mathrm{bs}^{2}$, C. B. Bridges, Nov. 24 , '25. RK 2 ; $\mathrm{bs}^{2} / \mathrm{Px}$ below $19^{\circ}$, RK 1.)

bw-brown. G. H. M. Waaler, Oct. 15, '19. 2-105.0. Pub. 1921. Eye-color light brownish pink, brilliant and translucent. Testes colorless. Malphigian tubules nearly colorless (. classifiable in larvae). Reoccurs with quite high frequency; also numerous allels. RK 1.

hy-humpy. C. B. Bridges, Oct. 22, '18. 2-93.3. Unpublished. Thorax strongly ridged, with "commas" at front and 2 pairs deep vortices. Wings obliquely truncated to half length. Highly sterile in both sexes. Viability low and erratic. Classification easy (but px no good in hy). RK 2 (location strategic).

ll-lanceolate. C. B. Bridges, Apr, 3, '23. 2-106.7士 (order of 11 and mr not determined). Unpublished. Wings narrowed at the tip and slightly divergent. RK 4. (More extreme allel $=11$, C. B. Bridges, Apr. 25, '23; RK 2).

INS-lethal-Nova Scotia. C. B. Bridges, Oct. 31, '25. 2-107.0-to right of l2ax, to left of $\mathrm{sp}$ ). Unpublished. Lethal in larval stage at $2 \mathrm{~mm}$ length, by weak and abnormal development of trachae and other chitinized parts. Present only in Inversion-Nova Scotia $\mathrm{px}$ sp stock. RK 4.

12ax-(lx)-lethal-2ax. C. B. Bridges, Feb. 28, '19. 2-106.9. Unpublished. Lethal in very early larva stage. RK 4.

M33a-(M33)-Minute-33a. J. Schultz, Jan. 7, '33. 2-108.0. Unpublished. From X-rayed o" Minute-bristles of "slight" type, with excellent viability, fertility and classifiability. Hom. = lethal. (Originally called M115). RK 1. Salivary analysis by Bridges shows deficiency of six 
lines, starting to right of heavy doublet $60 \mathrm{E} 1$ and ending to left of diff use doublet which is the last line of subsection $60 \mathrm{E}$.

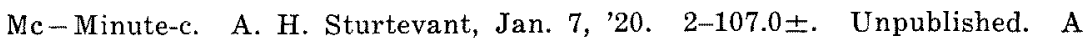
rather extreme "minute-bristle" type. Lost.

M]-Minute-1”. J. Schultz, Jan. 7, '26. 2-101.2. Unpublished. Minutebristles of slight type, with excellent viability, fertility and classifiability. Hom, = lethal. Not deficient for any known gene. RK 2. (In some relations RK 1) Salivary banding not detectedly abnormal.

mi-minus. R. L. Biddle, Dec. '28. 2-104.7. Unpublished. Bristles nearly as small as hairs. Hairs smaller and fewer. Body size very small. Emergence delayed. Viability low and erratic. Females entirely sterile, males fertile. RK 2-RK 4 .

$\mathrm{mr}$-morula. C. B. Bridges, Mar. 8, '13. 2-106.7 (11-mr order undetermined). Pub. Bridges and Morgan 1919; plate 10, figs. $3 \mathrm{a}$ and $3 \mathrm{~b}$. Eye rough. Bristles irregularly reduced in size and number. Abdominal sclerites smaller. Females entirely sterile, with undeveloped ovaries. RK 2. ( $\mathrm{mr}^{2}$, less extreme allel, C. B. Bridges, Nov. 24, '25).

pd-purpleoid. C. B. Bridges, Aug. 31, '16. 2-106.4. Unpublished. Eyecolor dark pink or maroon; like "purple" but less extreme. Semidominant, giving softening of eye color. Separation of hom. from het. by lighter orange "fleck". RK 2 .

pkh-pinkish. C. B. Bridges, July 27, '14. 2-106 \pm 5. Pub. Jour. Exp. Zool. $28: 337-384$. Specific dilutor of w-eosin. RK 4. Lost.

px-plexus. C. B. Bridges, Aug. 20, '14. 2-100.5. Pub. Bridges and Morgan 1919: text-fig. 80. Venation network of extra veins, especially toward tip and margin of wing. One of the most useful of the second-chromosome characters. RK 1.

sp-speck. T. H. Morgan, March '10. 2-107.0. Pub. Bridges and Morgan 1919 ; text-figs. $73 \mathrm{a}, 73 \mathrm{~b}, 75 \mathrm{~b}$, plate 5 , figs. 1, 2. Intense black speck in axil of wing. Body color darker, "olive". Best character in right cnd of 2. RK 1. (More intense allel $=\mathrm{sp}^{\prime}$; C. B. Bridges, June '25; RK 1).

\section{The banding of the salivary chromosomes}

That the salivary chromosomes are differentiated along their lengths by a succession of crossbands is well known. In aceto-carmine preparations the chromosome presents the picture of a faintly staining cylinder with strongly staining crossbands. The crossbands differ from each other markedly in intensity of stain, in the width of the bands, and also in character as a solid cross line or as a line broken into segments or dots. Most lines are sharply defined and hardedged, while some are diffuse and hazy of outline. In any given place in each chromosome the set of bands is characteristic and constant. However, different treatments in making the preparations (such as variation in the amount of iron, and differences in the length of staining and quality of the carmine itself) cause the relative intensities to appear somewhat different from preparation to preparation. 
That the chromosome is differentiated into two distinct substances is plain, not only from the staining or non-staining of specific points but from differences in refractive index of these same parts, whereby in fresh cells the same succession of high-refractive cross bands and low-refractive matrix, or substance between bands, can be seen. Also, under mechanical stress the dark-staining, highly refractive bands reveal themselves as solid, inelastic and disc-like, while the material between bands is very elastic-drawing out to at least three times its lax length without apparent disruptive change in its structure. Finally, the Fuelgen reaction shows that the solid, inelastic, highly refractive, darkly staining cross bands are highly charged with nucleic acid while the inter-band regions are relatively free of nucleic acid.

Intensive microscopical study of the banding has given us maps of the characteristic banding throughout the chromosomes (Painter 1934, 1935; Bridges 1935). In the bottom of Fig. 1 is given a map for the extreme right end of chromosome $2 \mathrm{R}$-corresponding to the part whose linkage map has already been described. This map is revised from that given in Bridges 1935 by a more complete presentation of the faint bands and by a more accurate representation of the specific characteristics of all bands.

The division of many crossbands into dots or segments is an expression of the multiple structure of the giant chromosome as a bundle of chromonemal threads derived through successive divisions of the original threads contributed by the gametes. Usually 16 of these granules can be seen in the bands of Drosophila melanogaster "diploid" chromosomes. Where fewer can be seen it is believed the threads are nevertheless divided but are still adherent by virtue of the synaptic action of the homologous parts of the threads. Because of this property of continued synaptic adhesion it may very well be that the visual number of threads is much lower than the actual number, very possibly 256 per haploid strand, as suggested by Hertwig (G. Hertwig 1935).

It would seem that there is a special class of bands (illustrated by $60 \mathrm{E} 1$ ) that is characterized by greater intensity than the majority of bands. Generally these "heavy" bands are clearly doublets, of which both lines have the same high intensity (illustrated by 60D1). They also often form "capsules" by union at their edges, with a clear space between $(58 \mathrm{~A}, 59 \mathrm{E})$. But even the medium and faint lines seem to be clearly doublets in the great majority of cases $(60 \mathrm{~A})$. What the significance of this predominant feature of the banding may be, is problematical. It could be a primary characteristic of band (and gene) structure or it could be a secondary doubled condition, brought 


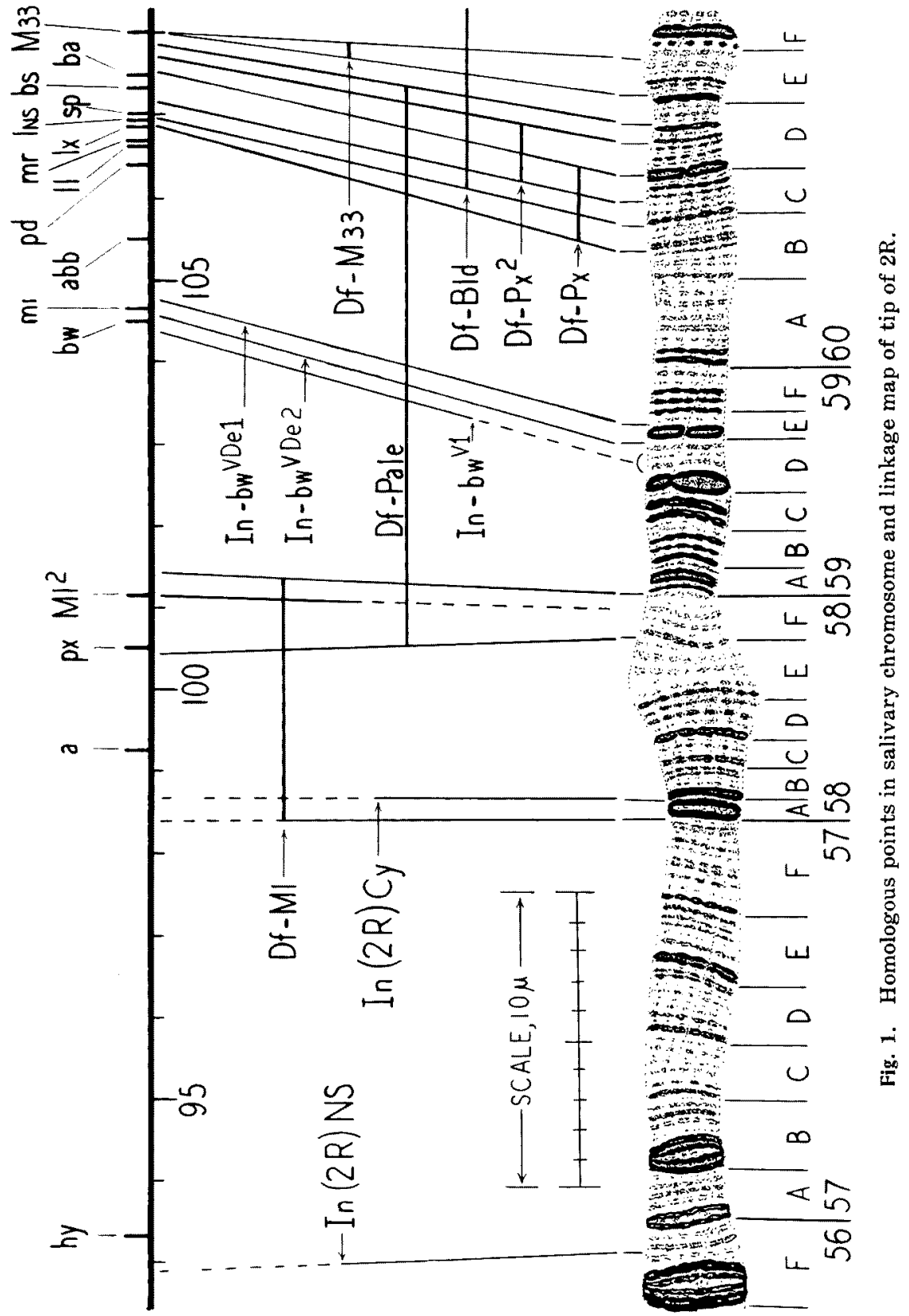

about, for example, by unequal crossing-over. The probabilities seem fair that the very heavy type of band is to be interpreted as secondary doubling. 
As pointed out before (Bridges 1935) there are sections of the chromosomes which in appearance are duplicates of other sections. In $56 \mathrm{~F}$ and $57 \mathrm{~B}$ are short groups of heavy bands which seem to be symmetrical reversed "repeats," that is, to consist of the same bands extending in both directions from a central point of symmetry. Furthermore it would seem that these two heavy groups are themselves repeats of each other-that the center of symmetry of this longer reversed repeat is in 57A. Another region suggestive of reversed repetition is in $58 \mathrm{E}-\mathrm{F}$.

Sections 58E and $\mathrm{F}$ show another characteristic peculiaritynamely, they often are converted into a much swollen light-staining "puff" in which the banding is very hard to see. The light region in the rounded tip of the chromosome (in 60E) also tends in this direction. Thus, sections of the chromosome show variations in the matrix or back-ground structure, as well as in the banding itself.

\section{The chromosomal aberrations and their use in homolo- gyzing the linkage with the salivary maps}

The most useful category of aberrations for homologyzing the two kinds of chromosome maps is deficiencies-the loss of a section of genes. The location of the deficiency on the linkage map can be found by the ordinary linkage tests, since the heterozygous deficiency usually gives somatic alterations constituting a dominant phenotype. Thus, the locus of the deficiency giving rise to "plexate" venation can be located as at a point some 2.4 units to the right of the locus brown and near the extreme tip of $2 \mathrm{R}$. It has been found that deficiencies give relatively very little alteration in the crossing-over frequencies and hence the linkage tests are in themselves very useful when properly interpreted. But for deficiencies there is another and very exact method of location, namely, the pseudo-dominance test for included loci. To illustrate this: the cross of Plexate by brown gives equal numbers of Plexate and of wild-type offspring, showing no pseudo-dominance of brown in the Plexate flies, but the cross of Plexate to speck yields wild-type offspring and Plexate offspring which also show speck. Since speck is a strict recessive this pseudodominance means that the deficiency has removed the wild-type allel of speck and that in Plexate flies the character speck is manifest for the same reason that the character yellow shows in the male with its single X-chromosome. With Plexate not only speck but also blistered and balloon and a lethal (INS) prove to be included in the deficiency. Thus, a section of the map from 106.9 to 107.5 and including 4 loci is known to correspond to the deficiency. Examination of the salivary gland chromosomes of the Plexate heterozygote 
shows that a section of about a dozen cross lines (counting doublets as 2) is absent. This section extends from line 60B7 (see lower line of Fig. 1) to the right of line 60D1. The break at the right end of the deficiency passes between the two equal lines of the heavy doublet. This fact shows that the parts of heavy doublets can have independent existence and hence can be each a gene.

The other deficiencies studied similarly were Plexate ${ }^{2}$, Minute$33 \mathrm{a}$ and two deficiencies derived from translocations, viz. the deficiency from $\mathrm{T}(2-3)$-Pale and from $\mathrm{T}(1-2)$-Blond. The limits of these deficiencies on the genetic and on the salivary map are indicated in Fig. 1. The dominant Minute- $\mathrm{l}^{2}$, which had been suspected of being a deficiency, has not thus far been found to have any bands missing.

The inversions do not offer very favorable material for homologizing the two maps, because crossing-over is so reduced in the presence of a heterozygous inversion that it is very difficult to determine where the break comes in the linkage map. But the establishment of the breakage point in the salivary chromosome is easy. Two of the first-found inversions have break-points in this region of $2 \mathrm{R}$, namely In (2R) -Nova Scotia and In (2R)-Curly. The salivary studies by Bridges and $\mathrm{Li}$ (unpublished) have shown these points to be as indicated in Fig. 1.

Schultz has studied the break-points of inversions associated with three of the variegated-brown dominants, namely, bwv1 ("Plum" of Muller) and two brown-variegates found by Demerec in the progeny of X-rayed flies. The locations of these breaks in the salivary map are given by Schultz in the lower line of Fig. 1. The breaks of these three inversions (In-bw $\mathrm{V}^{\mathrm{1}}$ has not as yet been closely determined) come in a narrow region in $59 \mathrm{D}-\mathrm{E}$. The brown-variegates from Demerec enable the locus of minus to be placed to the right of brown and in the heavy capsular doublet in $59 \mathrm{E}$.

For the loci INS, sp, bs and ba, the limits within which the given gene must lie are determined with fairly high accuracy and narrow range.

\section{Coefficients of crossing-over}

Comparison of the relative positions of given break-points in the two series shows clearly that the seriation is the same in both. However, the relative spacings vary somewhat according to the particular region. The studies of Painter and Bridges showed that the over-all lengths of the different salivary strands (X, 2L, 2R, 3L, 3R and 4) are closely proportional to the lengths of the corresponding linkage maps. We may define as the "normal" ratio of crossing-over this ratio of lengths of linkage maps to lengths of salivary chromosomes. The maps total 66, 108, 105 and 0.2 units, or approximately 279 units. 
The salivary strands total $1180 \mu$ (Bridges 1935). Hence the "normal" ratio is $1180 / 279=4.2 \mu$ per unit of map distance. Any section of chromosome can be said to have the normal coefficient of crossing-over (one) if it has $4.2 \mu$ of salivary map (as measured off on the maps of Bridges 1935) to one unit of crossing-over.

Regional differences in coefficient of crossing-over are strikingly apparent when $2 \mathrm{R}$ is examined, and are seen in the tip region. Thus, the map distance from $\mathrm{px}$ to $\mathrm{M} 33 \mathrm{a}$ is 7.5 units, corresponding to $18.5 \mu$. The ratio of crossing-over is thus $2.5 \mu$ per unit of crossingover, and the coefficient of crossing-over $(4.2 / 2.4)$ is 1.7 . That is, in this tip region, crossing-over is approximately $70 \%$ freer than in the set of chromosomes as a whole.

There are also local differences within this tip region. Thus, the px-bw map distance is 4.0 units, corresponding to $7 \mu$, giving a ratio of 1 unit $1.75 \mu$ and a very high coefficient of 2.4 .

\section{Coefficients of mutation}

A striking feature of the linkage maps is the bunching of mutant loci in certain regions, coupled with the occurrence of long stretches elsewhere relatively free from mutant loci. In the past such local differences have been explained by appeal to local differences in ease of crossing-over-for example, by the assumption of a low coefficient of crossing-over for the regions near the middle of chromosomes 2 and 3 . The salivary technique offers a better check on this than did the metaphase groups and it is seen that part of this non-random grouping is indeed due to differences in coefficient of crossing-over. However, the dense group of mutant loci at the end of $2 \mathrm{R}$ would be explained by an exceptionally low coefficient of crossing-over for that region, while we have just seen that the contrary is true-that for this tip region from $\mathrm{px}$ to $\mathrm{M} 33 \mathrm{a}$ crossing-over is about $70 \%$ above standard.

Another variable must be appealed to, namely the coefficient of mutation. It was only through such salivary studies as the present one that accurate enough information on the localization of mutants in the actual chromosome has been obtained to make it certain that regional differences in mutability exist. In Fig. 1, it can be seen that from the locus of humpy to plexus, is 7.2 units of map distance, corresponding to about $19 \mu$ of chromosome. Similarly, from px to M33a is 7.5 units of map distance corresponding to $18.5 \mu$. That is, the coefficients of crossing-over in these two sections are substantially equal (1.6 and 1.7). But, in the first section are only three mutant loci, while in the second are 15 . Since the included lengths of chromosome (together with numbers of crossbands) in these two sec- 
tions are substantially equal, we must attribute this striking difference to a mutability of genes approximately five times as high in the second section as in the first. A standard coefficient of mutability may be taken as the total of mutant loci divided into the total length of chromosomes. According to the latest revision of maps by Bridges (DIS-3:21-26) the number of loci of working mutants (probably proportional to the totals of all mutants) is about 280.

Hence the standard incidence of detected workable mutations is at present 1 mutant locus per $4.2 \mu$ of salivary length. The ratio of mutants to salivary length in the hy-px section is $3: 19$ or $1: 6.3$, and in the px-M33a section is $15: 18.5$ or $1: 1.2$. That is, in the hy-px section mutability is about $0.67 \times$ standard, while in the px-M33a section it is $3.5 \times$ standard.

It should be noted that the coefficient of mutation varies independently from the coefficient of crossing-over. This is readily seen in the two sections just compared, having the same somewhat high coefficient of crossing-over but very different coefficients of mutationone somewhat lower than normal and the other much higher. Furthermore, the data on which the two measures are based are different in nature-one depending on frequencies of crossing-over between given points and the other on merely the numbers of mutant loci between points.

\section{The interpretation of a low coefficient of detected mutation as a high frequency of duplications or "repeats"}

That the genes of a particular large section of chromosome should differ intrinsically in respect to mutability from those of the general group seems less likely than that secondary effects of some kind are present making regional differences. A regional factor which may very well account for most of the differences detected in the tip of $2 \mathrm{R}$ is the prevalence of duplications ("repeats") present in the normal chromosomes. The basal half of $2 \mathrm{~L}$ is very rich indeed with such duplications, and is also low in coefficient of mutation, considering that the locus of black is (according to unpublished data of Painter) at a point roughly one-third of the distance out toward the tip of $2 \mathrm{~L}$. If the genes of a given short section are present in another place in the group (either immediately adjacent or elsewhere in the same chromosome or in a different chormosome) then this duplication serves as a buffer against the manifestation of recessive mutants with loci in the given section. A recessive has to occur not only in that section but also in the duplication before it can be manifest. Hence any region rich in sections duplicated elsewhere will have a low coefficient of mutation. Even the incidence of dominants should be 
lowered, since in normal diploids many dominants give somatic effects only slightly above the threshold of detection and since the class of dominants from deficiencies would be reduced or absent. Observationally there seems to be a "reversed repeat", extending from the beginning of $56 \mathrm{~F}$ into the middle of $57 \mathrm{~B}$, and another in the puff of $58 \mathrm{E}$.

\section{Literature cited}

Bridges, C. B., 1935. Salivary chromosome maps, with a key to the banding of the chromosomes of Drosophila melanogaster. Jour. Hered. 26 : 60-64.

- 1935. Linkage maps. Drosoplita Information Service 3: 21-26.

Hertwig, G., 1935. Die Vielwertigkeit der Speicheldrüsenkerne und Chromosomen bei Drosophila melanogaster. Z.i.A.V., 70: 496-501.

Painter, T. S., 1933. A new method for the study of chromosome rearrangements and the plotting of chromosome maps. Science 78:585-586.

- 1934. Salivary chromosomes and the attack on the gene. Jour. Hered. 25: 464-476.

- 1935. The morphology of the third chromosome in the salivary gland of Lrosophila melanogaster and a new cytological map of this element. Genetics 20: $301-326$. 